Proc. Biomedical Simulation, 4th International Symposium, ISBMS 2008, Bello, F. and Edwards, E. (Eds.)

Lecture Notes in Computer Science, Vol. 5104, Springer Verlag, pp. 166-175.

\title{
Estimation of the Fracture Toughness of Soft Tissue From Needle Insertion
}

\author{
Toufic Azar and Vincent Hayward \\ Center for Intelligent Machines, McGill University \\ Montreal, Quebec, H3A 2A7, Canada, \\ toufic.azar@mail.mcgill.ca
}

\begin{abstract}
A fracture mechanics approach was employed to develop a model that can predict the penetration force during quasi-static needle insertion in soft tissue. The model captures a mechanical process where the sharp needle produces a crack that is opened to accommodate the shaft of the needle. This process involves the interchange of energy between four distinct phenomena: the work done by the needle, the irreversible work of fracture, the work of friction, and the change in recoverable strain energy. From measurements made in vivo, porcine liver fracture toughness was estimated from the difference in penetration force between two consecutive insertions at the same location. The values obtained fall within a reasonable range and confirm the relevance of a computational model of needle insertion based on fracture mechanics.
\end{abstract}

Key words: Needle Force, Fracture Mechanics, Fracture Toughness.

\section{Introduction}

Needle insertion is extensively used in medical intervention. Its use has expanded to a variety of minimally invasive and percutaneous procedures such as biopsies, neurosurgery and brachytherapy [1]. For pre-intervention planning, for intraintervention guidance, for post-intervention assessment, as well as for simulation purposes, a model of the physics of needle interaction with tissues during insertion would be valuable.

Here, we discuss a model that accounts for both the tissue and needle characteristics. Using in vivo measurements of needle insertion in pig liver performed by Maurin et al. [13], we could estimate the work of fracture and determine the liver fracture toughness. These values were found by comparing the response of the organ to a first penetration with the response of the same organ at the pre-cracked location. We found values that are in line with others cited in the literature and that were obtained using different methods.

The model that we developed can be used as a basis for several types of simulation. It can be used in a finite element analysis to find deflection from loading. Conversely, these values can be used to predict forces as a response to displacement for planning and simulation. 
Proc. Biomedical Simulation, 4th International Symposium, ISBMS 2008, Bello, F. and Edwards, E. (Eds.) Lecture Notes in Computer Science, Vol. 5104, Springer Verlag, pp. 166-175.

\section{Related Work}

Roughly speaking, needle insertion models fall under two categories. In the first category, needle penetration is viewed mostly from the view point to the deformation of tissues that the interaction causes. These models represent the effect of crack formation but do not actually model it because they do not account for the underlying physics of the insertion process. Instead, stiffness-like functions are fit to experimental data. These models cannot be invariant with respect to the choice of units. In the second category, models are based on the description of the mechanics of crack formation. The effects of a needle geometry interacting with tissue properties are responsible for the permanent changes made to an organ at the scale of cells and membranes. Despite these complexities, needle piercing and cutting create new surfaces inside a body, that is, damage. In the second category, damage is modeled by averaging over surfaces and through time. They are therefore based on energy methods.

Deformation models and function fit. Maurel represent the penetration force resulting from deformation as an exponential function of the depth [12], while Brett et al. use viscoelastic functions [5]. Simone and Okamura describe the needle force as the sum of the cutting force, the stiffness force and the friction force [15]. The stiffness-like model is equated to a second-order polynomial fitted to the data. Maurin et al. and Barbé et al. obtained fits using various functions [3, 4,13 . Another example of this approach is Dimaio and Salcudean's FEM models to determine the tissue deformation [7]. Recently, Hing et al. measured internal tissue deformation during needle penetration [10]. Since these techniques cannot represent combinations of tissue types and needle geometries, each case requires the determination of a new set of arbitrary parameters.

Energy models. Based on the work of Atkins [2], a multi-phase model suitable for the prediction of cutting forces was introduced by Mahvash and Hayward using energy and fracture mechanics [11]. The force of cutting is modeled as the result of an interchange of energy between the internal strain energy stored in the body due to initial deformation, the work done by the tool and the irreversible fracture work. Cutting and piercing both create new surfaces in a body. Therefore it should be possible to apply a fracture energy approach to needle insertion. In this view, the geometry that determines the cutting force is the size of the created surfaces. One may imagine that a needle causes a simple expansion to a cylindrical cavity. Shergold and Fleck portrayed the phenomenon as a modeI crack propagation with elastic deformation to accommodate the cylindrical shaft which suggests different crack geometries $[17,18]$. These geometries were observed in human skin and rubber samples. Using conservation of energy and neglecting friction, they determined the penetration pressure and crack size as a function of sharp-tipped punch diameters and tissue characteristics. Besides crack geometry, the second key parameter is tissue specific resistance to fracture. Authors have devised many types of experiments to determine it. 
Proc. Biomedical Simulation, 4th International Symposium, ISBMS 2008, Bello, F. and Edwards, E. (Eds.) Lecture Notes in Computer Science, Vol. 5104, Springer Verlag, pp. 166-175.

Testing approaches (Mode-I). Atkins used a guillotine-test to estimate the fracture toughness of skin and other soft tissue [2]. Mahvash and Hayward, robotically cutting potato and liver, as well as Doran et al., cutting animal skin, assumed that during the cutting phase the work of the tool was entirely transfered to the formation of the crack $[8,11]$. Pereira et al. used scissor cutting to obtain fracture toughness [16]. Chanthasopeephan et al. used scalpel cutting of liver [6]; the tool work was obtained from integration of the force-displacement curve. McCarthy et al. tackled the case of scalpel cutting where the tool is in permanent contact with the tissue [14]. Because friction was not be neglected, the work of fracture was equated to the work of the difference in cutting force between two consecutive insertions at the same location.

\section{$3 \quad$ Needle Insertion Phases}

Needle insertion and retraction at constant velocity experimentally suggests that the process can be decomposed into phases [11,13]. With reference to Fig. 1, four phases can be identified:

Phase 1: Deformation. The needle tip comes into contact with the tissue and deforms it without penetration. It terminates with puncture.

Phase 2: Steady-state penetration. Once an energy threshold is reached, the liver capsule ruptures and a crack initiates. Then, steady-state insertion initiates and the force increases with depth. This phase terminates when the needle stops.

Phase 3: Relaxation. When the desired depth is reached the motion is stopped and the force relaxes due to the viscoelastic properties of the material. The stored strain energy combined with friction creates a suction effect.

Phase 4: Extraction phase. The needle force is due to the friction force and the release of the stored elastic strain energy.

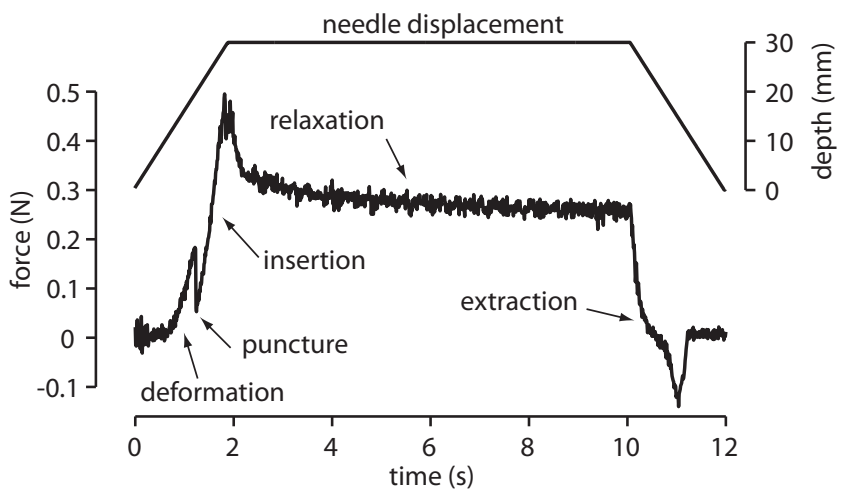

Fig. 1. Phases of needle insertion. This data, used with permission, is from [13]. 
Proc. Biomedical Simulation, 4th International Symposium, ISBMS 2008, Bello, F. and Edwards, E. (Eds.) Lecture Notes in Computer Science, Vol. 5104, Springer Verlag, pp. 166-175.

\subsection{Mechanism of Needle Insertion Into Liver}

Shergold et al. hypothethized and verified that the insertion of a sharp punch into skin and rubber causes the formation and wedging open of a mode-I crack, see Fig. 2 [18].
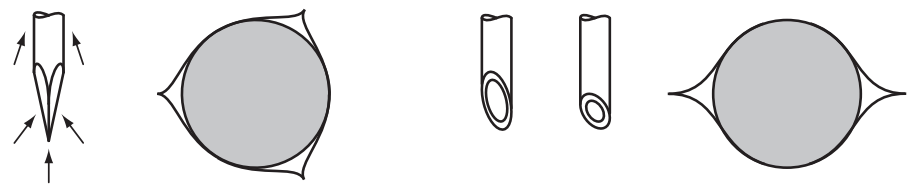

Fig. 2. Left: Geometry of a Franseen needle tip, forces acting on it and expected resulting 3-way branching or star-cracks. Right: standard $22^{\circ}$ bevel tip and $45^{\circ}$ bevel tip biopsy needles next to the expected resulting line crack.

To verify this theory, we obtained porcine liver samples directly from the slaughter house less than one day postmortem (refrigerated). The samples were manually penetrated with different needles types: (1) Franseen tip suture needle of diameter $0.84 \mathrm{~mm}$; (2) sharp standard bevel tip syringe needles of diameter $0.71 \mathrm{~mm}, 1.27 \mathrm{~mm}$, and $2.1 \mathrm{~mm}\left(22^{\circ}\right.$ bevel $)$; (3) true short bevel tip liver biopsy needle ( $45^{\circ}$ bevel tip) of diameter $1.47 \mathrm{~mm}$. The needles were dipped in trypan blue stain before insertion in an effort to increase image contrast. The samples were prepared with thin blades and the average size of the samples was $5 \times 5 \times 3 \mathrm{~mm}$. The cracks were examined using a binocular inverted microscope under $\times 10$ magnification. The cracks were measured at different depths, capsule level, $3 \mathrm{~mm}$ depth and $6 \mathrm{~mm}$ depth whenever it was possible to observe them. Fig. 3 shows representative examples of the images obtained. In the figures, images were processed using Photoshop ${ }^{\circledR}$, first discarding color information and then precisely bracketing the brightness levels to enhance contrast.

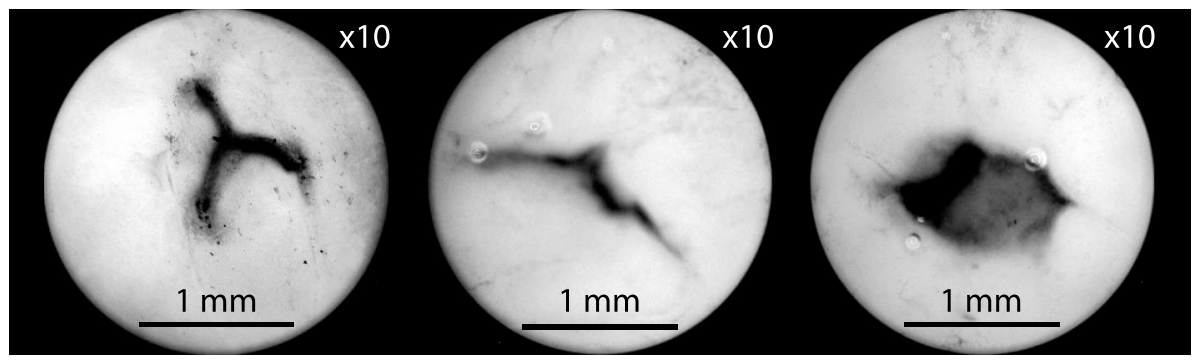

Fig. 3. Microscopic observations of fresh porcine liver penetrated with three types of needles. The left image shows the crack geometry made by a Franseen tip, the middle image show a crack resulting from a standard bevel tip, and the right image the crack left by a short bevel biopsy needle. 
Proc. Biomedical Simulation, 4th International Symposium, ISBMS 2008, Bello, F. and Edwards, E. (Eds.) Lecture Notes in Computer Science, Vol. 5104, Springer Verlag, pp. 166-175.

Fig. 4 shows cracks made by standard bevel needles with three different diameter. Cases where unstable crack formation was observed, that is, when the assumption of a sharp interaction breaks down were not considered. Unstable crack formation can be attributed to the non-homogeneity of the liver or unpredictable stresses induced by manual insertion.

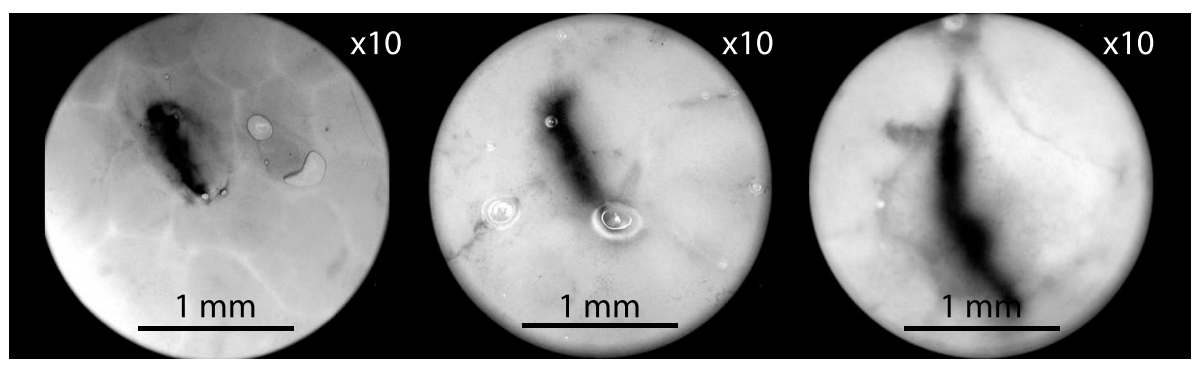

Fig. 4. Observations of fresh porcine liver penetrated with standard bevel needles of different diameters. From left to right, diameters of $0.71 \mathrm{~mm}, 1.27 \mathrm{~mm}$, and $2.10 \mathrm{~mm}$.

Table 1 collects a summary of the resulting crack sizes as a function of needle geometries. Only averages as shown as the sample size is too small to allow for statistical analysis.

Table 1. Crack length as a function of needle geometry (number of observations).

\begin{tabular}{lccccr}
\hline needle tip & $\begin{array}{c}\text { diameter } \\
(\mathrm{mm})\end{array}$ & $\begin{array}{c}\text { mean crack length } \\
\text { at capsule level } \\
(\mathrm{mm})\end{array}$ & $\begin{array}{c}\text { standard } \\
\text { deviation } \\
\text { (nb. observed) }\end{array}$ & $\begin{array}{c}\text { additional observations } \\
\text { (nb. observed) }\end{array}$ \\
\hline Franseen & 0.84 & 1.32 & $0.10(9)$ & $1.25(6)$ & $1.2(3)$ \\
$22^{\circ}$ bevel & 0.71 & 0.70 & $-(5)$ & & \\
$22^{\circ}$ bevel & 1.27 & 1.31 & $0.11(13)$ & $1.16(3)$ & \\
$22^{\circ}$ bevel & 2.10 & 1.93 & $-(3)$ & & \\
$45^{\circ}$ bevel & 1.47 & 1.70 & $0.28(6)$ &
\end{tabular}

From this preliminary study, despite the small sample size, it can be tentatively concluded that under the conditions of reasonable tissue homogeneity and needle sharpness, the diameter of a needle and the geometry of its tip are good predictors of both geometry and size of mode-I cracks. It can also be concluded that depth has little effect on crack size.

From our small sample set, we can predict a branch crack to be roughly 1.5 times larger than needle diameter that caused it. For sharp bevel tips, the crack length is about the same as the diameter of the needle, but for less sharp, short bevel, biopsy needles, as might be expected, the crack size is larger than the needle diameter which accounts for a larger insertion force. 
Proc. Biomedical Simulation, 4th International Symposium, ISBMS 2008, Bello, F. and Edwards, E. (Eds.) Lecture Notes in Computer Science, Vol. 5104, Springer Verlag, pp. 166-175.

\section{Fracture Mechanics Insertion Model}

When a sharp object penetrates a tissue, a crack propagates at its tip. An energy balance between two consecutive states assumes the following.

1. Elastic fracture. The deformation is plastic only at the vicinity of the crack.

2. Quasi-static process. Kinetic energy can be neglected, the velocity is low enough for the system to be in equilibrium at all times.

3. Sharp needle interaction. The tip is always in contact with the crack.

4. Constant crack width.

Combining the results in $[2,8,14]$, the energy balance equation gives

$$
F \mathrm{~d} u+\mathrm{d} U_{\mathrm{i}}=J_{\mathrm{IC}} \mathrm{d} A+\mathrm{d} \Delta+\mathrm{d} \Gamma+P \mathrm{~d} u .
$$

In this expression

- $F \mathrm{~d} u$ is the work done by the needle insertion force $F$ for an increment $\mathrm{d} u$,

- $U_{\mathrm{i}}$ is the internal strain energy stored in the material before indentation,

- $J_{\mathrm{IC}} \mathrm{d} A$ is the irreversible work of fracture in mode-I and $J_{\mathrm{IC}}$ is the critical fracture toughness (this lumps the different microscopic irreversibilities observed at the tip into a general constant that is material and mode specific).

$-\mathrm{d} A$ is the increment in crack area; for a line mode-I crack of width $a$, $\mathrm{d} A=a \mathrm{~d} u^{\prime}$ where $\mathrm{d} u^{\prime}$ is the crack depth, and $\mathrm{d} \Delta$ is the change in the stored internal recoverable strain energy potential. For instance, during scalpel cutting, the two created surfaces are free and the only strain energy stored is due to the deformation of the tissue in the direction of the cut. On the other hand, during the needle piercing phase, the surface created is also under transverse compression; with a plane-strain assumption, used in Shergold's model [18], the strain energy is stored proportionally to the insertion depth and vertical deformation subsequent to the initial deformation is neglected.

- $P \mathrm{~d} u$ is the work done by the friction force $P$ along the shaft of the needle. Its magnitude depends on the relative velocity between the needle and the tissue. In a quasi-static insertion, with the constant increase in contact area between the needle and the tissue, $P$ is proportional to the insertion depth.

$-\mathrm{d} \Gamma$ represents the work absorbed in plastic flow. This term is negligeable and is ignored [18]; we assume that once the needle is extracted the body takes back its original geometry and size.

We now apply (1) to the different phases of an insertion. Referring to Fig. 5:

Phase 1 deformation: The needle work creates a deformation $\delta_{i}$. Assuming that the tissue is initially at rest (1), becomes

$$
F \mathrm{~d} u=\mathrm{d} \Delta .
$$

This force may always be represented as polynomial function $F(\delta)$. This interaction reduces to a contact mechanics problem between a tool and a substrate and for which numerical and analytical solution exists. 
Proc. Biomedical Simulation, 4th International Symposium, ISBMS 2008, Bello, F. and Edwards, E. (Eds.) Lecture Notes in Computer Science, Vol. 5104, Springer Verlag, pp. 166-175.
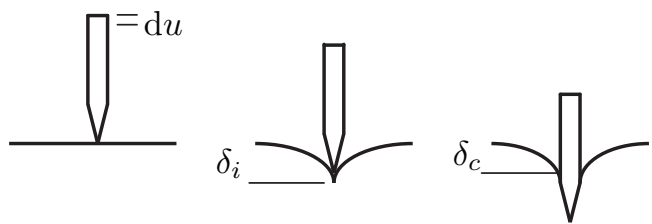

Fig. 5. Definition of displacement quantities.

Phase 1.5 rupture: When rupture occurs a crack propagates. The maximal deformation $\delta_{i}$ relaxes to $\delta_{c}$ (Fig. 5). During this short instant, the force exhibits a sharp decrease in magnitude and a crack of depth $\delta_{i}-\delta_{c}$ is formed. Heverly et al. showed that the force required to initiate cutting reduces with increasing needle velocity, up to a critical speed above which, the rate independent cutting force of the underlying tissue becomes the limiting factor [9]. At this critical rate the tissue deformation is minimal. The limiting velocity is one order of magnitude larger than the data obtained from [13].

Phase 2 penetration: The true crack depth is $u^{\prime}=u-\delta_{c}$. Since the tool displacement is equal to the crack propagation, the energy equation becomes

$$
F \mathrm{~d} u=J_{\mathrm{IC}} \mathrm{d} A+\mathrm{d} \Delta+P \mathrm{~d} u,
$$

where $d \Delta$, the work per unit length to wedge open the crack, could be calculated using deformation models, by considering an expanding wedge of radius, $r$, from $r=0$ to $R$ [18]. In Shergold and Fleck's model the crack width is determined as a function of $R, J_{\mathrm{IC}}$ and the tissue properties, shear modulus $\mu$ and strain hardening $\alpha$. Assumptions include plane strain, isotropic, non-viscous and incompressible material. Since their model ignores friction, the predicted crack length is probably overestimated.

\section{Fracture Toughness Determination}

To determine the work of fracture, $F_{c} \mathrm{~d} a$, where $F_{c}$ is the cutting force, an approach similar to that in $[14,16]$ is employed. A cutting-pass penetration of the needle is first carried out. During insertion, the friction, wedging and fracture work, all contribute to the force $F$. The energy balance remains that of (3). A second penetration is performed at the same location. All phenomena are nearly identical except for the energy required to create the crack, which vanishes. This gives a smaller needle force $F^{\prime}$ and (3) reduces to

$$
F^{\prime} \mathrm{d} u=\mathrm{d} \Delta+P \mathrm{~d} u .
$$

Subtracting (3) from (4) and noting that $\mathrm{d} u^{\prime}=\mathrm{d} u$ we obtain an explicit relationship for the fracture toughness

$$
\left(F-F^{\prime}\right) \mathrm{d} u=J_{\mathrm{IC}} a \mathrm{~d} u .
$$


Proc. Biomedical Simulation, 4th International Symposium, ISBMS 2008, Bello, F. and Edwards, E. (Eds.) Lecture Notes in Computer Science, Vol. 5104, Springer Verlag, pp. 166-175.

Evaluating $\int_{u_{0}}^{u_{1}}\left(F-F^{\prime}\right) \mathrm{d} u$ as a function of $\mathrm{d} A=a \mathrm{~d} u$ where $u_{0}$ is the onset of the steady penetration phase and $u_{i}$ is when it terminates. The slope of the estimated curve is the fracture toughness $J_{\mathrm{IC}}$ of the pierced tissue. See Fig. 6 .
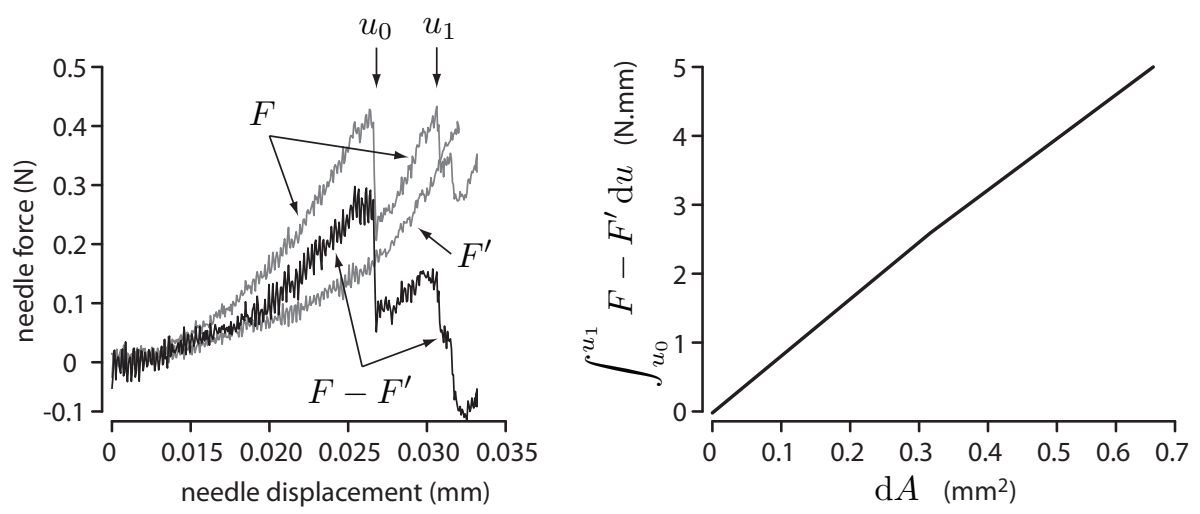

Fig. 6. Representative example of the determination of $J_{\mathrm{IC}}$ using integration.

\section{Results}

In Maurin et al. [13], an 18 gauge, $1.27 \mathrm{~mm}$ diameter, $22^{\circ}$ biopsy needle was roboticaly inserted in vivo into a pig liver at different slow velocities with a pause of $8 \mathrm{~s}$ between the insertion and the extraction phases. During insertion, forces and displacements were recorded.

We applied the model described in the previous section to their data. Based on our in vitro experiments of Section 3.1, the crack length was estimated to be equal to the needle diameter. This assumption is realistic since the difference in tissue condition is expected to marginally affect the crack size.

For each of the four measurements, labeled as in the original reference, the integral $\int_{u_{0}}^{u_{1}} F_{n}-F_{n}^{\prime} \mathrm{d} u$ was obtained using the trapezoidal approximation and plotted against the crack area expansion. The slopes obtained are collected in Table 2.

Table 2. Fracture toughness for different measurements.

\begin{tabular}{ccc}
\hline Measurements & $J_{\mathrm{IC}}\left(\mathrm{J} \cdot \mathrm{m}^{-2}\right)$ & Velocity $\left(\mathrm{mm} \cdot \mathrm{s}^{-1}\right)$ \\
\hline 3b & 75.8 & 15 \\
4b & 95.7 & 15 \\
5b & 91.8 & 6 \\
6b & 185.6 & 19 \\
\hline
\end{tabular}


Proc. Biomedical Simulation, 4th International Symposium, ISBMS 2008, Bello, F. and Edwards, E. (Eds.) Lecture Notes in Computer Science, Vol. 5104, Springer Verlag, pp. 166-175.

Fracture Toughness From Needle Insertion

\section{Discussion}

Measurements $3 \mathrm{~b}, 4 \mathrm{~b}$ and $5 \mathrm{~b}$ fall within a narrow range of values while measurement $6 \mathrm{~b}$ gave a higher value. Such disparity cannot be attributed to the change in velocity but rather to inhomogeneity of the organ. The number of measurements is not statistically significant, but the range of fracture toughness is reasonable 76 to $185 \mathrm{~J} \cdot \mathrm{m}^{-2}$. Additional tests would likely confirm these results. By comparison, Chanthasopeephan et al. found for a dead pig liver, using scalpel cutting, a fracture toughness ranging from 187 to $225 \mathrm{~J} \cdot \mathrm{m}^{-2}$ [6]. This discrepancy is easily explained. Scalpel-liver interactions differ from needle insertion. Differences may also exist in the sharpness of the tool; a sharper tool produces less blunting [14]. The test conditions were also different. Maurin et al.'s experiments were done in vivo with both breathing and non-breathing animals.

The accuracy of ours results is relative to our assumptions and to method employed. The crack width could only be estimated. A $10 \%$ error in the crack size estimate would result in a $10 \%$ error in fracture toughness. Plastic deformation was also neglected but this assumption is reasonable. When a free-pass was run, it was assumed that the needle experienced the same friction forces and caused the same strain energy storage as a piercing-cut. Data from multiple passes at a same location should be collected to investigate this effect further.

In future experiments, tissue deformation and friction should be modeled, and in combination with fracture mechanics, analytical needle tip forces should be compared to experimental results. In addition, other experimental conditions should be controlled and varied. For example, the introduction of kinetic energy to account for non-steady insertions or taking into account the viscosity of the tissue would certainly improve the model.

\section{Conclusion}

Needle insertion was modeled as controlled crack propagation. We first confirmed that a planar crack is created when a biopsy needle is inserted into liver and that a star-crack results from using a Franseen tip. By using an energy approach, the fracture toughness of porcine liver was estimated. Our technique can be applied for other needle geometries and tissues. A significant variable is the crack size which can reflect the tip geometry and can be set to simulate a variety of needles. Future work should combine these results with FEM deformation models.

\section{Acknowledgments}

The authors wish to thank Benjamin Maurin and Laurent Barbé for sharing their data as well as Thomas Steffen and Janet Moir for help with imaging the samples. This article is based on "Estimation of the penetration force of needle insertion into soft tissue" submitted in partial fulfillment of course "Haptics" taught by the second author at McGill University. The second author would like to acknowledge the support of NSERC, the Natural Sciences and Engineering Research Council of Canada for a Discovery grant. 
Proc. Biomedical Simulation, 4th International Symposium, ISBMS 2008, Bello, F. and Edwards, E. (Eds.) Lecture Notes in Computer Science, Vol. 5104, Springer Verlag, pp. 166-175.

\section{References}

1. Abolhassani, N., Patel, R., Moallem, M.: Needle Insertion Into Soft Tissue: a Survey. Med. Eng. \& Phy. 29, 413-431 (2007)

2. Atkins, A. G., Mai, Y.-W.: Elastic and Plastic Fracture, Metals, Polymers, Ceramics, Composites, Biological Materials. Ellis Horwood Limited (1985)

3. Barbé, L., Bayle, B., Mathelin, M., Gangi, A.: Needle Insertions modeling: Identifiability and limitations. Biomed. Sig. Process. Contr. 2, 191-198 (2007)

4. Barbé, L., Bayle, B., Mathelin, M., Gangi, A.:In Vivo Model Estimation and Haptic Characterization of Needle Insertions. Int. J. Robotics Research 26, pp. 1283-1300 (2007)

5. Brett, P. N., Parker, T. J., Harrisson, A. J., Thomas, T. A.: Simulation of Resistance Forces Acting on Surgical Needles. Proceedings of the Institution of Mechanical Engineering, vol. 211, 335-345 (1997)

6. Chanthasopeephan, T., Desai, J. P., Lau, A. C. W.: Determining Fracture Characteristics in Scalpel Cutting of Soft Tissue. IEEE/RAS-EMBS Conference on Biomedical Robotics and Biomechatronics, 899-904 (2006)

7. Dimaio, S. P., Salcudean, S. E.: Needle Insertion Modeling and Simulation. IEEE T. on Robotics and Automation 19, 191-198 (2003)

8. Doran, C. F., McCormack, B. A. O., Macey, A.: A Simplified Model to Determine the Contribution of Strain Energy in the Failure Process of Thin Biological Membrane During Cutting. Strain 40, 173-179 (2004)

9. Heverly, M., Dupont, P.: Trajectory Optimization for Dynamic Needle Insertion. IEEE Int. Conf. on Robotics and Automation, 1646-1651 (2005)

10. Hing, J. T., Brooks, A. D., Desai, J. P.: A biplanar uoroscopic approach for the measurement, modeling, and simulation of needle and soft-tissue interaction. Medical Image Analysis 11, 62-78 (2007)

11. Mahvash , M., Hayward, V.: Haptic Rendering of Cutting: a Fracture Mechanics Approach. Haptics-e 2, (2001)

12. Maurel, W.: 3D Modeling of the Human Upper Limb Including the Biomechanics of Joints, Muscles and Soft Tissues. PhD thesis, Laboratoire d'Infographie, Ecole Polytechnique Federale de Lausanne, (1999)

13. Maurin, B., Barbe, L., Bayle, B., Zanne, P., Gangloff, J., Mathelin, M., Gangi, A., Soler, L., Forgione, A.: In Vivo Study of Forces during Needle Insertions. Perspective in Image-guided Surgery: Proceedings of the Scientific Workshop on Medical Robotics, Navigation, and Visualization (MRNV'04), pp. 415-422, (2004)

14. McCarthy, C. T., Hussey, M., Gilchrist, M. D.: On the Sharpness of Straight Edge Blades in Cuting Soft Solids: Part I - Identation Experiments. Eng. Fract. Mech. 74, 2205-2224 (2007)

15. Okamura, A. M., Simone, C., O'leary, M. D.: Force Modeling for Needle Insertion into Soft Tissue. IEEE T. on Biomedical Engineering 51, 1707-1716 (2004)

16. Pereira, B. P., Lucas, P. W., Teoh, S.-H.: Ranking the Fracture toughness of Thins Mammalian soft Tissues Using the Scissors Cutting Test. J. Biomechanics 30, 91-94 (1997)

17. Shergold, O. A., Fleck, N. A.: Mechanisms of Deep Penetration of Soft Solids, With Application to the Injection and Wounding of Skin. Procedings Royal Society London, Vol. A 460, 3037-3058 (2004)

18. Shergold, O. A., Fleck, N. A.: Experimental Investigation Into the Deep Penetration of Soft Solids by Sharp and Blunt Punches With Application to the Piercing of Skin. J. Biomechanical Eng. 127, 838-848 (2005) 